\title{
MtWOX9-1 gene as somatic embryogenesis stimulator. Search of targets
}

\author{
Varvara Tvorogova \\ SPSU, St Petersburg, Russia \\ krubaza@mail.ru \\ Ksenia Kuznetsova \\ SPSU, St Petersburg, Russia \\ kskuz95@mail.ru
}

\author{
Elizaveta Krasnoperova \\ SPSU, St Petersburg, Russia \\ liza_krasnoperova99@mail.ru \\ Elina Potsenkovskaya \\ SPSU, St Petersburg, Russia \\ epots556@gmail.com
}

\author{
Andrei Kudriashov \\ SPSU, St Petersburg, Russia \\ a23.10.2012@yandex.ru \\ Ludmila Lutova \\ SPSU, St Petersburg, Russia \\ la.lutova@gmail.com
}

\begin{abstract}
MtWOX9-1 gene was shown to stimulate somatic embryogenesis in vitro in Medicago truncatula. To understand its functions in this process, we performed transcriptomic analysis of $M t W O X 9-1$ overexpressing calli, as well as expression correlation analysis. As a result, we obtained a list of possible MtWOX9-1 targets during somatic emyryogenesis. Now we are checking these possible targets using ChIP-seq analysis.
\end{abstract}

Keywords - somatic embryogenesis, transcription factors, Medicago truncatula

Motivation and Aim

\section{Motivation}

Somatic embryogenesis (SE) is the development of embryo-like structures from somatic plant tissues. SE in vitro is widely used for plant propagation and transformation; therefore, the search for SE stimulators and revealing of the mechanisms of their functioning are very important for biotechnology.

\section{Aim}

Previously, we have shown that transcription factor MtWOX9-1, belonging to the WOX family, can stimulate SE in the Medicago truncatula callus culture. In this research, transcriptomic analysis of highly embryogenic calli with MtWOX9-1 overexpression was performed.

\section{Methods}

We used RNA from transgenic calli with MtWOX9-1 overexpression and wildtype calli of R-108 line as a control to perform trancriptomic analysis. During bioinformatics processing of transcriptomic data, we used Trimmomatic for deleting adapter sequences (Bolger et al., 2014), HISAT2 for alignment with the reference genome (Kim et al., 2015), Stringtie for counting reads (Pertea et al., 2015), DESeq2 for the search for differentially expressed genes (Love et al., 2014), GSEABase for gene enrichment analysis (Morgan et al., 2019) and WGCNA for gene correlation networks search (Langfelder, Horvath, 2008).

\section{Results}

It was shown that MtWOX9-1 overexpression led to the activation of several groups of genes, including genes related to cell division, tissue differentiation, and seed development. Among enriched GO groups, we found several gene sets, related with repressive epigenetic changes. Using Medicago Truncatula Gene Expression Atlas, we also identified a group of genes encoding for transcription factors, that were both coexpressed with MtWOX9-1 in different plant organs and differentially expressed in our samples. These genes are putative targets of MtWOX9-1, and they may act in the same pathway with this regulator during SE. Now we are checking, if MtWOX9-1 binds indeed to these targets, using ChIP-seq analysis.

\section{ACKNOWLEDGMENT}

Supported by the RFBR (19-14-50209, 20-016-00124) and RSF (16-16-10011).

\section{REFERENCES}

[1] Bolger A. M., Lohse M., Usadel B. (2014) Trimmomatic: a flexible trimmer for Illumina sequence data. Bioinformatics 30(15): 2114-2120

[2] Kim D., Langmead B., Salzberg S. L. (2015) HISAT: a fast spliced aligner with low memory requirements. Nature Methods 12(4): 357360.

[3] Langfelder P., Horvath S. (2008) WGCNA: an R package for weighted correlation network analysis. BMC Bioinformatics 9(1): 559.

[4] Love M. I., Huber W., Anders S. (2014) Moderated estimation of fold change and dispersion for RNA-seq data with DESeq2. Genome Biol 15(12):550.

[5] Morgan M., Falcon S., Gentleman R. (2019). GSEABase: Gene set enrichment data structures and methods. R package version 1.48.0.

[6] Pertea, M. et al. (2015) StringTie enables improved reconstruction of a transcriptome from RNA-seq reads. Nat Biotechnol 33(3): 290-295. 